\title{
USO DE LAS TIC DESPIERTA UNA MAYOR MOTIVACIÓN QUE CON LA NO INCLUSIÓN DE LAS MISMAS EN EL PROCESO DE ENSEÑANZA Y APRENDIZAJE
}

\author{
USE OF TIC TAKES A GREATER MOTIVATION THAT WITH THE \\ NONINCLUSION OF THE SAME IN THE TEACHING AND LEARNING \\ PROCESS
}

\author{
${ }^{\mathrm{a}}$ Martha Catalina Ospina Hernández, ${ }^{\mathrm{b}}$ Albert Miyer Suarez, ${ }^{\mathrm{c}}$ Gloria Patricia Espinosa Morales \\ ${ }^{\mathrm{d}}$ Nohora Smith Jaimes Sandoval \\ ${ }^{a}$ Universidad Francisco de Paula Santander Ocaña, Grupo de Investigación INGAP, Vía \\ Acolsure Sede el Algodonal, Ocaña - Norte de Santander, Colombia, dwricob@ufpso.edu.co \\ ${ }^{\mathrm{b}}$ Universidad de Pamplona, Grupo de Investigación Gimup, Pamplona Colombia, \\ albertmiyer@unipmaplona.edu.co
}

Fecha de recepción: 05-07-2015

Fecha de aprobación: 10-03-2016

\begin{abstract}
Resumen: Este artículo es una síntesis de resultados de una investigación cualitativa. Se analiza cómo el uso de las Tecnologías de la Información y Comunicación (TIC) despierta, en estudiantes del grado noveno de básica secundaria, una motivación mayor que la que muestran estudiantes en cuyo proceso de enseñanza y aprendizaje estas no se incluyen. La información se recogió en cuatro instituciones educativas urbanas de Colombia tales como Bogotá, Medellín, Floridablanca y Cali, mediante dos cuestionarios de preguntas sencillas y de fácil comprensión aplicados a veinte estudiantes y ocho docentes. En el análisis de resultados se aplicó la Triangulación para darle al proceso mayor precisión y profundidad (Vallejo y Finol 2009). Se determinaron tres categorías: Competencias digitales, las TIC en el ambiente educativo, y uso pedagógico de las TIC. Se obtuvieron los siguientes resultados: las nuevas herramientas tecnológicas dinamizan el proceso de enseñanza y aprendizaje, propician ambientes pedagógicos más acogedores y motivadores, y permiten al docente presentar los contenidos de una manera más dinámica e interactiva que la empleada en la instrucción tradicional. Se concluye que: los docentes reconocen la importancia de la inclusión de las TIC en la educación, y necesitan mayor capacitación en el uso de estas herramientas; los estudiantes conocen y utilizan un mayor número de herramientas tecnológicas, y disfrutan incorporándolas en sus procesos de aprendizaje y en la realización de actividades escolares; y tanto profesores como estudiantes señalan la deficiente dotación de herramientas tecnológicas en las aulas.
\end{abstract}

Palabras clave: Tecnologías de la Información y Comunicación (TIC), motivación, enseñanza, aprendizaje y Triangulación. 


\begin{abstract}
This article is a digest of the results of a qualitative research. It analyzes how the use of technologies of information and communication (ICT) stimulates in ninth graders a motivation greater than that of students whose teaching and learning process does not include ICT. The information was gathered in four urban educational institutions in Colombia such as Bogotá, Medellín, Floridablanca and Cali, through two questionnaires containing simple questions easy to understand, applied to twenty students and eight teachers. In the result analysis the principle of Triangulation method was applied in order to give the process greater precision and depth (Vallejo \& Finol, 2009). Three categories were determined: digital competence, ICT in the educational environment, and pedagogical use of ICT. The findings were: the new technological tools turn the teaching and learning process more dynamic, foster more comfortable and motivating pedagogical environments, and allow for teachers to present contents more dynamically and interactively than used in traditional instruction. It is concluded that teachers acknowledge the importance of including ICT in education, need more training in the use of those tools; students know and use a larger number of technological tools and enjoy incorporating them in their learning process and in doing their school activities; and both teachers and students point out the scarce provision of technological tools for classrooms.
\end{abstract}

Keywords: Information and Communication Technologies (ICT), motivation, teaching, learning and Triangulation.

\section{Introducción}

Los cambios y retos tecnológicos son necesarios en la escuela. Y uno de los objetivos que deben mantenerse en continuo crecimiento en la educación es el "desarrollar las aptitudes cognoscitivas y el carácter moral y ético que aseguraría, según el pensamiento sobresaliente de la época, ciudadanos capaces de participar en una sociedad más compleja, cada vez más industrializada, como miembros productivos, respetuosos de la ley y socialmente responsables" (Fullan y Stiegelbauer, 1997).

En esta línea de pensamiento, el aprendizaje significativo es clave en el proceso de enseñanza aprendizaje de educandos íntegros. Aquí, la motivación es una de las herramientas clave para el aprendizaje real. Ella impulsa a los estudiantes a su autoeficacia y confianza en sus capacidades para llevar a la práctica cualquier proyecto que se propongan: solucionar problemas, aprender de diferentes situaciones, realizar acciones de mejora para sí mismos y su entorno. En esta motivación de logro se presentan la fuerza del motivo, las expectativas y el valor de la tarea, elementos que marcan la disponibilidad de la persona hacia la excelencia (Heredia y Sánchez, 2012).

Por lo anterior, y porque se sabe que un ambiente interactivo favorece el aprendizaje autónomo, colaborativo y el pensamiento crítico (Domínguez, 2009), se vio la necesidad de observar y analizar los niveles motivacionales del estudiante en relación con las herramientas tecnológicas utilizadas por el docente en su quehacer pedagógico. 
En este ámbito, se encuentran varias investigaciones en diferentes contextos. Entre ellas está la de Alonso, J. (1992b), "Motivar en la adolescencia": Teoría, evaluación e intervención. Su trabajo se enfoca en 3 temas centrales: determinantes motivacionales del aprendizaje en la adolescencia, motivación e interacción en el aula, y estrategias para el cambio motivacional. Los resultados apuntan a la necesidad de introducir en el proyecto educativo objetivos de tipo motivacional claros. Es imprescindible definir las causas que hay que incluir y cómo hay que actuar para desarrollarlas.

También, están los aportes de Heredia y Sánchez (2012), quienes definen la motivación como el proceso de surgimiento, manteniendo la regulación de los actos que producen cambios en el ambiente. La motivación se presenta como una fuerza que impulsa a los individuos a tomar decisiones o realizar acciones y esta puede ser intrínseca (voluntaria) o extrínseca (ajena a la voluntad).

En la nueva sociedad de la información y la globalización, es de vital importancia indagar $\mathrm{y}$ aplicar nuevas herramientas $\mathrm{y}$ recursos en el proceso formativo que respalden totalmente el aprendizaje significativo de los estudiantes. Con base en lo anterior, los estudios realizados por Zenteno y Montera (2011) sobre la influencia de las Tic en la educación muestran cómo la tecnología suscita en los docentes y en los estudiantes cambios motivacionales, y en algunos ocasiones esto genera mejora en los procesos de aprendizaje. Similares resultados arrojan las investigaciones de Capacho, J. (2011), por un lado, y la de Viera, L. (2010), por otro. Esta última muestra los factores que determinan el uso de la tecnología en el contexto del desarrollo de estrategias desde la labor docente.

Las anteriores intervenciones inspiran una pregunta para la investigación: ¿El uso de las TIC despierta, en los estudiantes del grado noveno de las instituciones seleccionadas para el estudio, una mayor motivación que la que muestran con la no inclusión de las mismas en el proceso de enseñanza y aprendizaje?

A la luz de esta pregunta, el presente estudio se propone un objetivo general: determinar si las herramientas tecnológicas son motivantes para el proceso de enseñanza y aprendizaje en los estudiantes del grado noveno de educación básica secundaria; y tres objetivos específicos: establecer cuáles herramientas tecnológicas tienen mayor aceptación entre los estudiantes del grado noveno de educación básica secundaria en su proceso de enseñanza y aprendizaje; identificar el impacto que tiene el uso de las TIC sobre la motivación en los estudiantes $\mathrm{y}$, finalmente, reunir información acerca del acceso y manejo de herramientas tecnológicas de los estudiantes y docentes del grado noveno de educación básica secundaria en su proceso de enseñanza y aprendizaje.

\section{Metodología}

El enfoque utilizado para esta investigación fue el cualitativo. Con éste se pretendía dar una explicación sobre el nivel de motivación de los estudiantes al recibir sus clases utilizando las TIC. El proceso de recolección de datos y su análisis fue hecho todo por las investigadoras, quienes (como lo indica una de las propiedades de dicho enfoque), tenían un conocimiento previo 
sobre este tema, lo que también les permitía emitir juicios de lo observado para la investigación; debido a lo anterior, la investigación es, también, de tipo fenomenológico: "se enfoca en la experiencia subjetiva del individuo y en describir los significados de las experiencias vividas por las personas con respecto a un concepto o fenómeno"(Valenzuela y Flores, 2012, p.92).

Para la investigación se seleccionó una muestra finita y discreta de 8 docentes (dos por cada institución) del grado noveno, de diferentes contextos (ubicación, estrato, de carácter público y privado) y de diferentes áreas de enseñanza; igualmente, una muestra aleatoria y simple de 20 estudiantes (cinco por cada institución), del mismo grado, de los centros educativos donde laboran las investigadoras.

Debido a su enfoque cualitativo, para la recolección de datos se utilizaron dos cuestionarios: uno dirigido a los docentes, y otro, a los estudiantes, de modalidad autoadministrados. Dichos cuestionarios fueron diseñados por las investigadoras, teniendo en cuenta algunas variables establecidas en los objetivos de la investigación. Los estudiantes respondieron un cuestionario de 11 ítems, con preguntas cortas, claras y sencillas, de opción múltiple (Anexo A). Los docentes resolvieron un cuestionario de 15 ítems, con preguntas semiabiertas, igualmente sencillas y fáciles de contestar (Anexo B).

Para iniciar el proceso, se dio a conocer en cada una de las instituciones educativas donde se realizó la investigación, la propuesta en donde se plantearon los objetivos y pregunta problematizadora. Esto permitió generar un proceso de reconocimiento, compromiso y credibilidad frente al trabajo que se estaba realizando.

Posteriormente, se pasó a la recolección de datos con los estudiantes y docentes seleccionados para el estudio. Ellos, antes de contestar los cuestionarios, fueron informados del objetivo de la misma y, para mostrar su mayor validez, firmaron un formato de consentimiento en el que manifestaban que estaban de acuerdo con participar en la investigación de manera voluntaria.

Teniendo los resultados de las encuestas, se procedió al análisis de datos. Se clasificaron las respuestas en las siguientes categorías e indicadores que influyen en forma directa en la problemática de investigación:

Categoría A: Competencias digitales

1. Indicador: Definición e importancia de las TIC

\section{Indicador: Manejo de las TIC}

3. Indicador: Lugar en el que se hace uso de las TIC

4. Indicador: Habilidad en el uso de las TIC

Categoría B: Las TIC en el Ambiente Educativo

1. Indicador: Disponibilidad de las TIC 
2. Indicador: Aprovechamiento de las TIC

3. Indicador: Frecuencia del uso de las TIC

4. Indicador: Dificultad en el acceso a las TIC

Categoría C: Uso Pedagógico de las TIC

1. Indicador: Inclusión de las TIC en el proceso de enseñanza y aprendizaje

2. Indicador: Utilidad de las TIC en el proceso de aprendizaje

3. Indicador: Cambio de actitud y motivación con el uso de las TIC

4. Indicador: Desarrollo de material digital

Debido al enfoque cualitativo de la investigación y a los diferentes sujetos de estudio, el equipo se basó en la Triangulación, como técnica para dicho análisis. "Triangular significa dar un apoyo a un resultado, mostrando que éste coincide con otro" (Valenzuela y Flores, 2012, p. 195). Para ello, se revisaron los cuestionarios realizados a los estudiantes y a los docentes y se hizo la distribución de cada una de las preguntas en cada una de las categorías nombradas anteriormente.
Al realizar la Triangulación, se identificaron aquellos resultados que coincidían en los sujetos de estudio y que resultaban relevantes para dar respuesta a la pregunta planteada al inicio de la investigación: ¿El uso de las TIC despierta, en los estudiantes del grado noveno de las instituciones seleccionadas para el estudio, una mayor motivación que la que muestran con la no inclusión de las mismas en el proceso de enseñanza y aprendizaje? Además, se obtuvieron unos resultados más precisos, ya que "la integración de diferentes puntos de vista permite al proceso investigativo mayor precisión y profundidad", según Vallejo y Finol (2009, pp. 130-131).

\section{Resultados y discusión}

La presentación del producto obtenido en este trabajo de investigación está centrado en el análisis de los instrumentos diseñados para el estudio; los resultados arrojan información valiosa que permite dar respuesta a la pregunta problematizadora. En la descripción de las categorías se relacionan algunas gráficas enumeradas con base en los cuestionarios utilizados para la recolección de la información y que se exponen en los anexos.

\section{Categoría A: Competencia digitales}

En esta categoría los ítems abordados indagan sobre la definición e importancia de las TIC, su manejo, lugar en que se hace uso de ellas y la habilidad en su uso. Se evidencia que la población investigada (docentes y estudiantes del grado noveno $\left(9^{\circ}\right)$ de educación básica secundaria), tiene conocimiento y manejo de herramientas tecnológicas y le da relevancia a la inclusión 
de las mismas en el proceso de enseñanza y aprendizaje.

- $\quad$ En cuanto a los docentes: el $75 \%$ de los docentes tiene clara la definición de TIC e incluye estas herramientas en los procesos de enseñanza y aprendizaje; el $7.5 \%$ considera las TIC como una herramienta de apoyo alternativa para la enseñanza; el 75\% las ve como un factor determinante en el aprendizaje de los estudiantes; el $62.5 \%$ halla que las TIC promueven el interés y la motivación de los alumnos.

- Del grupo de estudiantes investigado, el $100 \%$ admite que es importante la incorporación de las TIC en la educación.

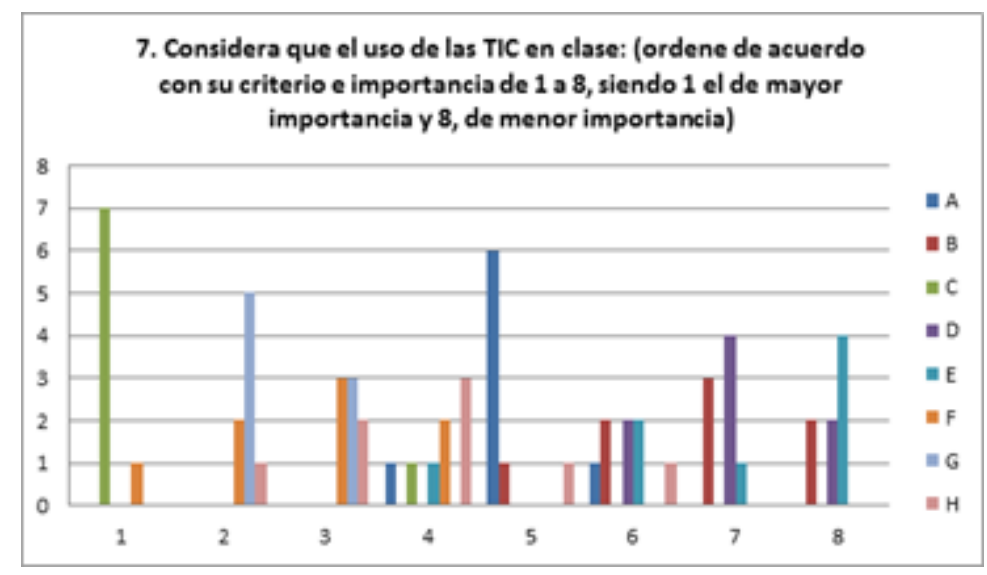

Figura 1. Datos recabados por las autoras.

Fuente: (Tomado de cuestionario para docentes)

- La mayoría de los estudiantes investigados expresa que hace uso de las TIC. Las herramientas más utilizadas son: en un $100 \%$, los computadores de mesa y computadores portátiles; en un $95 \%$, los dispositivos de almacenamiento, internet y correo electrónico. Los recursos que menos manejan son los foros, con un 50\%, y el software educativo, en un 55\%. También, un $45 \%$ menciona que hace una mayor utilización de estos recursos tecnológicos en la casa y en el colegio.

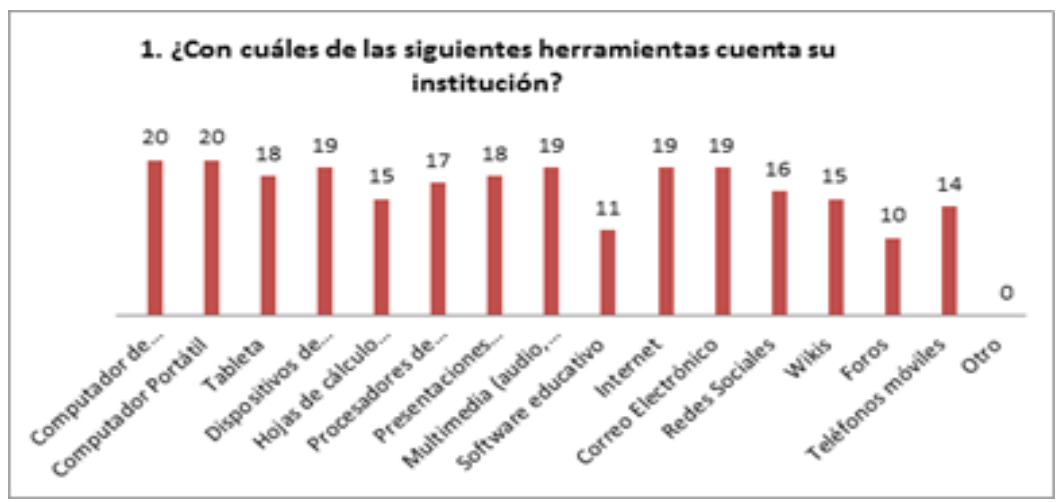


Figura 2. Datos recabados por las autoras.

Fuente: (Tomado de cuestionario para estudiantes)

- En cuanto a habilidades en el manejo de las TIC, la población docente manifiesta: el $50 \%$ tiene suficiente dominio; un $37.5 \%$, buen nivel y un $37.5 \%$ ha recibido una buena formación en el uso de TIC; pero a su vez, el $37.5 \%$ expresa que su formación en TIC ha sido nula.

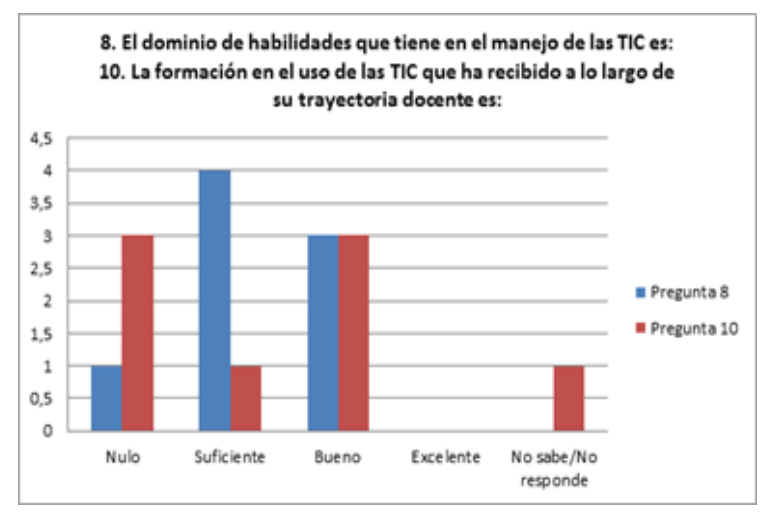

Figura 3 Datos recabados por las autoras.

Fuente: (Tomado de cuestionario para docentes)

\section{Categoría B: Las TIC en el ámbito educativo}

En esta categoría se tuvo en cuenta la disponibilidad que tienen de las TIC, su aprovechamiento, la frecuencia de su uso y las dificultades de acceso a ellas. Se evidencia que todavía existe una brecha entre los diversos recursos y herramientas tecnológicas y el proceso educativo.

- $\quad$ Respecto a la disponibilidad de TIC, los resultados muestran que tanto las instituciones educativas como un $62.5 \%$ de docentes cuentan con herramientas tecnológicas dentro del aula para el desarrollo de sus clases.

Los estudiantes manifiestan, frente a la inclusión de TIC en el proceso de enseñanza y aprendizaje, que las herramientas más empleadas son las presentaciones y el internet, en un $95 \%$; los computadores de mesa, las hojas de cálculo y los procesadores de texto, en un 80\%; los dispositivos de almacenamiento, en un $75 \%$. Las herramientas tecnológicas menos utilizadas en el proceso educativo son: la tableta, con un $35 \%$; las redes sociales, en un $20 \%$, y los teléfonos móviles, con un $15 \%$.

A pesar de que tanto docentes como estudiantes tienen disponibilidad y poseen un manejo de algunos recursos TIC, los docentes utilizan con poca constancia material didáctico digital para el desarrollo de sus clases: solo el 25\% lo usa frecuentemente, y el $37.5 \%$ lo emplea a veces.

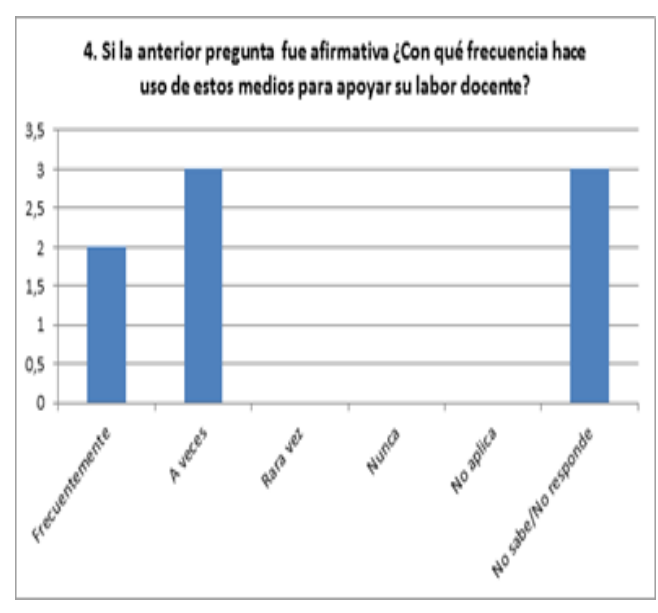

Figura 4. Datos recabados por las autoras. Fuente: (Tomado de cuestionario para docentes) 
- $\quad$ En cuanto al uso de herramientas TIC en la institución educativa, $60 \%$ de los estudiantes manifiesta que algunas veces hacen uso de ellas, y $37.5 \%$ de los docentes, a veces hacen uso de estos medios en su labor. Aquí se puede observar una gran diferencia entre docentes y estudiantes en cuanto a la frecuencia con la cual hacen uso de herramientas y recursos TIC.

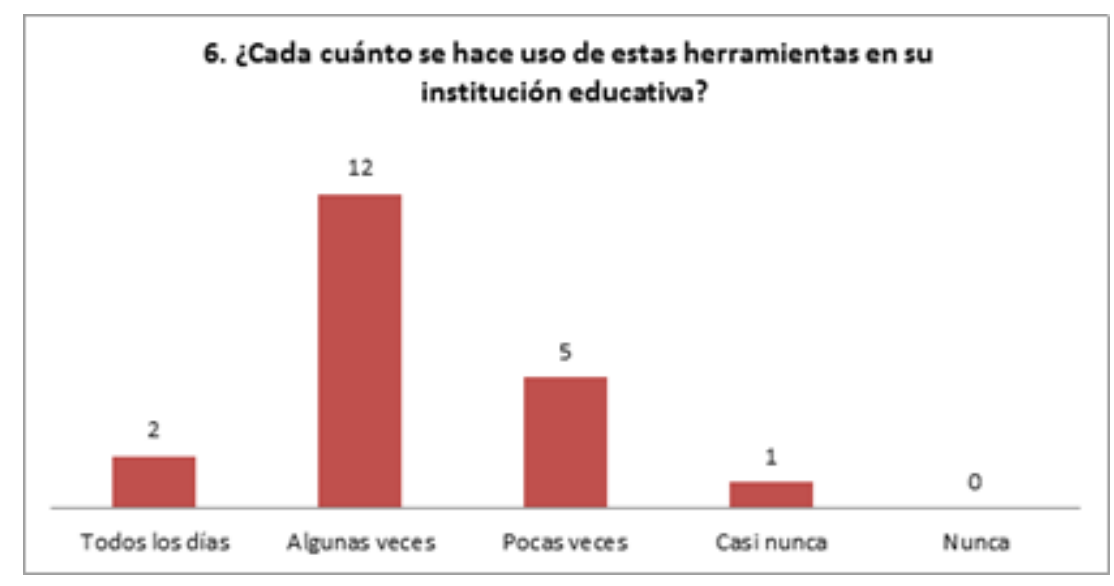

Figura 5. Datos recabados por las autoras.

Fuente: (Tomado de cuestionario para estudiantes)

- $\quad$ En cuanto al acceso a las TIC dentro de la institución educativa, el $75 \%$ de los estudiantes manifiesta que su mayor dificultad en el acceso a ellas está en el poco número de medios tecnológicos en relación con la demanda estudiantil.

\section{Categoría C: Uso pedagógico de las TIC}

En esta categoría se abordó la inclusión de las TIC en el proceso de enseñanza y aprendizaje, su utilidad en el proceso de aprendizaje, el cambio de actitud y motivación con el uso y desarrollo de material digital. Se observa que la mayoría de los docentes le da un uso significativo a las TIC con el fin de tener buenas prácticas pedagógicas.

- $\quad$ En cuanto a los estudiantes, el $70 \%$ considera que las herramientas tecnológicas son útiles; al 50\% le gusta aprender utilizando herramientas tecnológicas; el 45\% estima que mejora el rendimiento escolar cuando usa herramientas tecnológicas; el $40 \%$ sostiene que su grupo familiar está interesado en que ellos aprendan a utilizar estas herramientas y el 35\% expresa que aprende mejor cuando las usa en clases. 


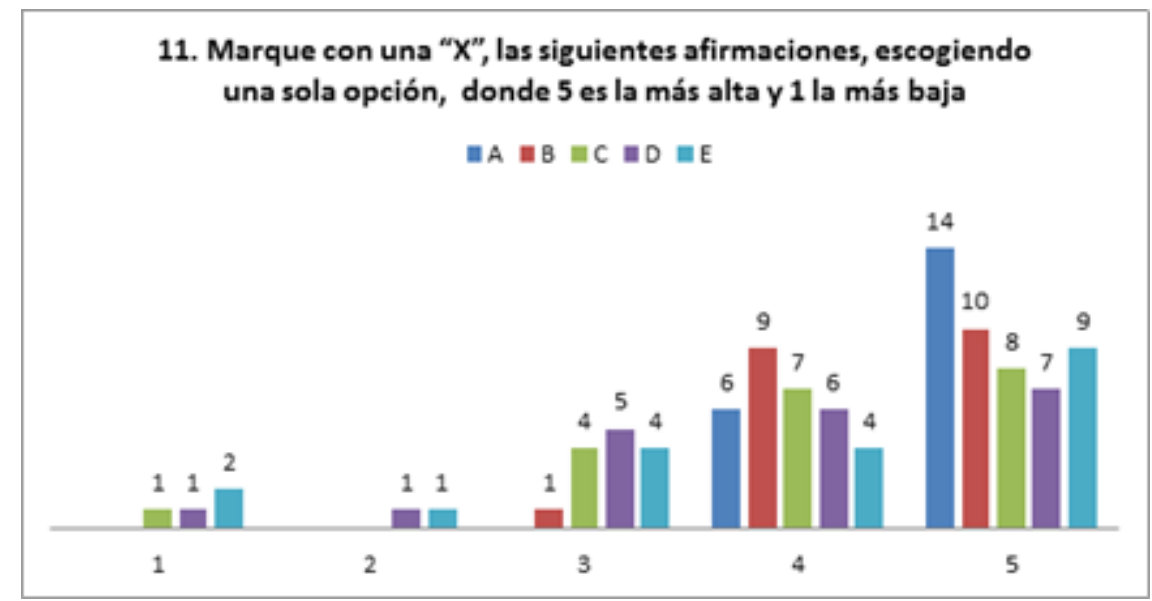

Figura 6. Datos recabados por las autoras.

Fuente: (Tomado de cuestionario para estudiantes)

- $\quad$ En cuanto a los docentes, el $62.5 \%$ emplea medios tecnológicos para sus clases y usa las nuevas tecnologías de la información y la comunicación para comunicarse con sus estudiantes; mientras que el $37.5 \%$ no incluye estos recursos en su quehacer pedagógico.
El $100 \%$ de la población investigada considera que la utilización de herramientas tecnológicas en el proceso de enseñanza y aprendizaje aumenta los niveles de interés y motivación.

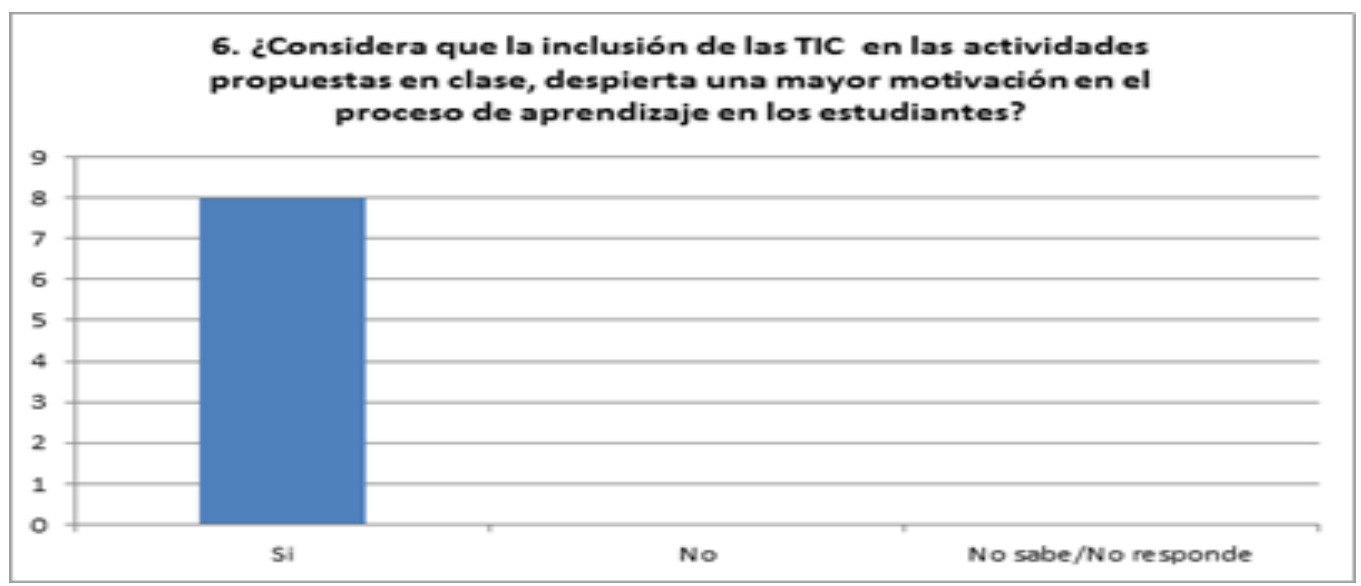

Figura 7. Datos recabados por las autoras.

Fuente: (Tomado de cuestionariopara docentes)

El $50 \%$ de los docentes ha generado material pedagógico utilizando recursos digitales; el $25 \%$ lo utiliza frecuentemente y a veces para el desarrollo de las clases.

\section{Conclusiones}

El siglo XXI ha alcanzado niveles fantásticos en la informática. Las nuevas 
generaciones van creciendo inmersas en ese mundo digital. En este contexto, la presente investigación muestra que su uso en el aula y fuera de ella motiva al $100 \%$ de los estudiantes para realizar un aprendizaje autónomo significativo. En contraste, aunque esta no fue incluida metodológicamente en el presente estudio, la observación directa de estudiantes en cuya instrucción no se hace uso de las herramientas de TIC permite notar, en muchos de estos, una mayor dispersión y negligencia para abordar su proceso de aprendizaje autónomo significativo.

De esta manera, se puede responder afirmativamente la pregunta central de esta investigación y la pregunta indirecta sobre el impacto de las TIC.

En cuanto a las TIC de mayor aceptación y uso entre los estudiantes encuestados, los computadores de mesa y portátiles son los preferidos, seguidos de cerca por Internet, dispositivos de almacenamiento de datos y el correo electrónico.

En cuanto al acceso y uso de las TIC dentro de las instituciones educativas, si bien existe un número de herramientas, no son suficientes en relación con el número de estudiantes. Esto reduce la posibilidad de acceso y uso por parte de ellos. Por otra parte, no todos los profesores están en condiciones de usar esas herramientas, principalmente por falta de capacitación.

De lo anterior se desprende la urgente necesidad de que las instituciones educativas sean suficientemente dotadas de herramientas de TIC, y que los profesores sean capacitados en su utilización.

\section{Referencias}

Alonso, J. (1992b). Motivar en la adolescencia: Teoría, evaluación e intervención. Madrid. 357 páginas.

Capacho, J. (2011). Evaluación del aprendizaje en espacios virtuales: Tic. Capítulo 1.3. Teorías del aprendizaje y su relación con las Tecnologías de la Información y las comunicaciones.

Domínguez, E. (2009). Las Tic como apoyo al desarrollo de los procesos de pensamiento y construcción activa de conocimientos. Zona Próxima, Revista del Instituto de estudios en educación Universidad del Norte. (19). 146-155

Fullan, M. y Stiegelbauer, S. (1997). Fuentes de Cambio en el cambio educativo. (pp.25-35). Editorial Trillas. Distrito Federal, México.

Heredia, Y. y Sánchez, A. (2012). Motivación. En Heredia, Y. y Sánchez, A.L. (Ed.), Teorías del aprendizaje en el contexto educativo (pp.154-163). Monterrey, México: Editorial digital Tecnológico de Monterrey.

Valenzuela, J. y Flores, M. (2012). El análisis de datos, los resultados y su discusión. En Fundamentos de la Investigación Educativa. 2. (p. 195). México.: Editorial Digital Tecnológico de Monterrey. 
apoyo a las funciones docente. Estado de México.

Vallejo, R. y Finol, M. (2009). La triangulación como procedimiento de análisis para investigaciones educativas. (pp. 130-131). Revista Electrónica de Humanidades, Educación y Comunicación Social. Maracaibo, Venezuela.

Zenteno, A., \& Montera, F. (2011). Integración y apropiación de las TIC en los profesores y los alumnos de educación media superior. Apertura: Revista de Innovación Educativa, 3(1), (pp. 1-10). México

Viera, L. (2010). Las tecnologías de información y comunicación como

Anexos

Anexo A

Cuestionario con preguntas de opción múltiple para estudiantes

Con el objeto de identificar las percepciones acerca del uso de la tecnología en el aula, el conocimiento de la misma en los profesores, y el impacto de la tecnología en el aprendizaje de los estudiantes; un equipo investigativo conformado por profesionales ha decidido iniciar una trabajo mediante el que se pueda determinar la calidad de los procesos relacionados con el uso y manejo de las TIC en la educación.

Una de las herramientas que el equipo investigativo está dispuesto a utilizar es la siguiente encuesta con preguntas de opción múltiple, el cual no genera ningún compromiso de parte del encuestado y no tiene ninguna repercusión de carácter legal sobre el mismo. Con el fin de que los datos obtenidos a partir del siguiente test sean lo más reales posibles, le solicitamos sea honesto (a) y sensato (a) a la hora de responder a cada uno de los planteamientos expuestos en este documento.

De antemano agradecemos su colaboración y disposición para responder este cuestionario.

Nombre:

Colegio:

Ciudad: Grado:

1. Señale con una "X", cuáles de los siguientes herramientas sabe utilizar (puede marcar más de una si es necesario). 

a. ( ) Computador de Escritorio
b. ( ) Computador Portátil
c. ( ) Tableta
d. ( ) Dispositivos de almacenamiento (USB. DVD, CD)
e. ( ) Hojas de cálculo (Lotus, Excel)
f. ( ) Procesadores de Texto (Block de notas, Word)
g. ( ) Presentaciones (PowerPoint)
h. ( ) Multimedia (audio, video)
i. ( ) Software educativo
j. $\quad(\quad)$ Internet
k. ( ) Correo Electrónico
1. ( ) Redes Sociales
m. ( ) Wiki
n. ( ) Foros - Videoconferencias
o. ( ) Teléfonos móviles
p. Otro (especifique)

2. Marque con una " $X$ " en qué lugar hace uso de esas herramientas (marque solo una respuesta)
a. ( ) En el colegio
b. ( ) En la casa
c. ( ) En la casa y el colegio
d. ( ) Fuera de la casa y el colegio
e. ( ) Otro (especifique)

3. Señale con una " $X$ ", con cuáles de las siguientes herramientas cuenta su institución (puede marcar más de una si es necesario)
a. ( ) Computador de Escritorio
b. ( ) Computador Portátil 

c. ( ) Tableta
d. ( ) Dispositivos de almacenamiento (USB. DVD, CD)
e. ( ) Hojas de cálculo (Lotus, Excel)
f. ( ) Procesadores de Texto (Block de notas, Word)
g. ( ) Presentaciones (PowerPoint)
h. ( ) Multimedia (audio, video)
i. ( ) Software educativo
j. $\quad(\quad)$ Internet
k. ( ) Correo Electrónico
1. ( ) Redes Sociales
m. ( ) Wiki
n. ( ) Foros- Videoconferencias
o. ( ) Teléfonos móviles
p. Otro (especifique)

4. Señale con una "X", cuáles de los siguientes herramientas son utilizadas para su proceso de enseñanza-aprendizaje (puede marcar más de una si es necesario).
a. ( ) Computador de Escritorio
b. ( ) Computador Portátil
c. ( ) Tableta
d. ( ) Dispositivos de almacenamiento (USB. DVD, CD)
e. ( ) Hojas de cálculo (Lotus, Excel)
f. ( ) Procesadores de Texto (Block de notas, Word)
g. ( ) Presentaciones (PowerPoint)
h. ( ) Multimedia (audio, video)
i. ( ) Software educativo
j. ( ) Internet
k. ( ) Correo Electrónico 
1. ( ) Redes Sociales

m. ( ) Wiki

n. ( ) Foros

o. ( ) Teléfonos móviles

p. Otro (especifique)

5. Marque con una "X", ¿Cuáles cree que son las principales dificultades que se pueden tener para acceder a estas herramientas en su institución educativa? (puede marcar más de una si es necesario).
a. ( ) Están dañados
b. ( ) Son muy pocos para el número de estudiantes que hay
c. ( Están en buen estado pero no los tienen funcionando
d. ( ) Las herramientas son obsoletas
e. ( ) No hay los espacios adecuados para el uso de esas herramientas
f. ( ) No hay ningún interés por hacer uso de ellas
g. Otro (especifique)

6. Marque con una "X", ¿cada cuánto se hace uso de estas herramientas en su institución educativa? (marque solo una respuesta)
a. ( ) Todos los días
b. ( ) Algunas veces
c. ( ) Pocas veces
d. ( ) Casi nunca
e. ( ) Nunca

7. Para usted, ¿es importante contar con la incorporación de las Tecnología de la información y Comunicación (TIC) en la educación? Sí No 
8. Para usted, ¿el uso de estas herramientas tecnológicas (TIC) ayuda a mejorar el nivel académico en los estudiantes? Sí No

9. Para usted, ¿las herramientas tecnológicas (TIC) deben ser utilizadas por todos los docentes en las diferentes áreas, para hacer más agradables las clases? Sí No

10. ¿Considera que la inclusión de las TIC en las actividades propuestas en clase, despierta una mayor motivación en el proceso de aprendizaje en los estudiantes? Sí No

11. Marque con una " $\mathrm{X}$ ", las siguientes afirmaciones, escogiendo una sola opción, donde 5 es la más alta y 1 la más baja

a. Usar las herramientas tecnológicas es útil.

1( ) 2( ) 3( ) 4( ) 5( )

b. Me gusta aprender utilizando las herramientas tecnológicas.

1( ) 2( ) 3( ) 4( ) 5( )

c. Mis padres están interesados en que aprenda a usar herramientas tecnológicas

1( ) $2($ ) $3($ ) $4($ ) $5($ )

d. Aprendo mejor cuando uso las herramientas tecnológicas en clase

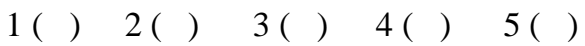

e. Mi rendimiento escolar mejora cuando hago uso de las herramientas tecnológicas
1( ) 2( ) 3( ) 4( ) 5( )

Muchas gracias por su cooperación, 
Grupo de Investigación

Anexo B

Formato de Encuesta/Cuestionario para docentes

Fecha de realización: Impreso: __ Encuesta postal:

Nombre del docente:

Institución Educativa: Grados que enseña: Asignatura:

Un grupo de investigadores conformados por profesionales de la educación desea averiguar el efecto motivador que despierta las tecnologías de la información y la comunicación (en adelante TIC) en los procesos de enseñanza-aprendizaje en los estudiantes. Esta investigación se inicia averiguando el nivel de conocimiento del uso de algunos recursos tecnológicos por parte de los docentes y sus apreciaciones de las TIC en la educación.

El instrumento que el grupo investigativo seleccionó es el cuestionario utilizado en la investigación por encuesta sugerido a partir de las lecturas de Valenzuela y Flores (2011). Se agradece al docente seleccionado su colaboración con el tiempo concedido para responder dicho documento y, se aclara que éste no tiene ninguna repercusión de carácter legal y además, se garantiza la confidencialidad. Su honestidad en el momento de responder este instrumento es la clave para llevar con éxito la investigación.

1. Para usted, profesor(a), ¿qué significan las Tecnologías de la Información y la Comunicación (TIC)?

a. Son tecnologías de la información y de comunicaciones, constan de equipos de programas informáticos y medios de comunicación para reunir, almacenar, procesar, transmitir y presentar información en cualquier formato, es decir, voz, datos, textos e imágenes.

b. Elementos y las técnicas usadas en el tratamiento y la transmisión de las informaciones, principalmente de informática, internet y telecomunicaciones.

c. a. $y$ b.

d. N__ Ninguna de las anteriores

e.Otras 
2. ¿Su salón cuenta con medios tecnológicos? Sí No

3. Si la anterior pregunta fue afirmativa ¿usa esos medios para sus clases?

Sí

No

4. Si la anterior pregunta fue afirmativa ¿Con qué frecuencia hace uso de estos medios para apoyar su labor docente? (marque con una X)

Frecuentemente A veces Rara vez Nunca No aplica

5. ¿De qué manera ha contribuido a mejorar el desarrollo de sus clases al utilizar los recursos tecnológicos, que la institución ha puesto a su disposición? (marque con una X)

Significativa Poco significativa No hay recursos tecnológicos en el aula

¿Por qué?

$6 \quad$ ¿Considera que la inclusión de las TIC en las actividades propuestas en clase, despierta una mayor motivación en el proceso de aprendizaje en los estudiantes?

Sí__ No_

¿Por qué?

7. Considera que el uso de las TIC en clase: (ordene de acuerdo con su criterio e importancia de 1 a 8 , siendo 1 el de mayor importancia y 8, de menor importancia) 
a. Es un factor determinante en el aprendizaje de los estudiantes

b. Es una moda dada de la era tecnológica en la que vivimos

c. Es una herramienta de apoyo alternativa para la enseñanza de los diversos contenidos

d. Es una herramienta totalmente prescindible

e. Es una alternativa que no necesariamente influye en el aprendizaje de los estudiantes

f. Es un recurso importante para mejorar la enseñanza

g. Promueve el interés y la motivación de sus alumnos

h. Facilita el trabajo en grupo y la colaboración con sus estudiantes

8. El dominio de habilidades que tiene en el manejo de las TIC es: (marque con una X)

Nulo Suficiente

Bueno

Excelente

9. La confianza que siente al emplear los medios tecnológicos frente al grupo es: (marque con una X) Buena Regular Baja

10. La formación en el uso de las TIC que ha recibido a lo largo de su trayectoria docente es: (marque con una $\mathrm{X}$ )

Insuficiente Suficiente Óptima Excesiva 
11. ¿Considera necesario cursos especiales de formación en el uso las TIC para los profesores? (marque con una $\mathrm{X}$ ) Sí No

12. ¿Considera necesario cursos especiales de formación en el uso las TIC para los estudiantes? (marque con una $\mathrm{X}$ ) Sí No

13. ¿Ha creado material didáctico digital para sus clases? (marque con una X)

Sí__ No

¿De qué tipo?

14. El material didáctico digital que usted ha desarrollado ha sido utilizado en el desarrollo de sus clases: (marque con una X)

Frecuentemente A veces Rara vez Nunca

15. ¿Utiliza usted las nuevas tecnologías para comunicarse con sus alumnos? (blogs, correo electrónico, chat, página personal, plataformas educativas...): (marque con una X)

Sí

No

Muchas gracias por su cooperación,

Grupo de Investigación 